\title{
Author Correction: Dystonia
}

Bettina Balint, Niccolò E. Mencacci, Enza Maria Valente, Antonio Pisani, John Rothwell, Joseph Jankovic, Marie Vidailhet and Kailash P. Bhatia

Nature Reviews Disease Primers 4, Article number: 25 (2018) https://doi.org/10.1038/ s41572-018-0023-6 Published online 20 September 2018

The affiliation for Enza Maria Valente and Antonio Pisani at IRCCS Santa Lucia Foundation, Rome, Italy, has been amended to remove the laboratory designation. Additionally, in Figure 3a, the subthalamic nucleus was incorrectly included in the anatomy of the brain shown and this has been amended.

https://doi.org/10.1038/s41572-018-0039-y I Published online: 19 October 2018 